\title{
Reexploring 5-methoxyindole- 2-carboxylic acid (MICA) as a potential antidiabetic agent
}

This article was published in the following Dove Press journal: Diabetes, Metabolic Syndrome and Obesity:Targets and Therapy

\author{
Liang-Jun Yan \\ Department of Pharmaceutical \\ Sciences, UNT System College of \\ Pharmacy, University of North Texas \\ Health Science Center, Fort Worth, \\ TX, USA
}

\begin{abstract}
Methoxyindole-2-carboxylic acid (MICA) is a potent hypoglycemic agent that inhibits gluconeogenesis in the liver. It is also a well-known inhibitor of mitochondrial dihydrolipoamide dehydrogenase. MICA was extensively studied in the 1960s and 1970s and was once tested for its antidiabetic effect in diabetic Chinese hamsters, whereby MICA was shown to exhibit pronounced glucose-lowering ability while also leading to increased rate of death of the diabetic animals. Since then, MICA's potential ability in lowering blood glucose in diabetes has never been revisited. In my opinion, MICA should be comprehensively reexplored for its antidiabetic properties in a variety of rodent diabetes models. For a given animal model, its dosedependent effect and the effects of different routes of administrations as well as its synergistic effects with other glucose-lowering drugs should also be investigated. More studies in the future on this chemical may provide novel insights into its role as an antidiabetic agent.
\end{abstract}

Keywords: 5-methoxyindole-2-carboxylic acid, antidiabetic, diabetes, dihydrolipoamide dehydrogenase, gluconeogenesis, mitochondria

\section{Introduction}

Diabetes mellitus, as an insidious disease, has become epidemic and is now a leading health issue globally. This disease is caused by dysregulation of glucose metabolism resulting in persistently high levels of blood glucose that imparts glucotoxicity. ${ }^{1,2}$ In adult-onset diabetes, also known as type 2 diabetes, development and progression of the disease is initiated by insulin resistance followed by $\beta$-cell dysfunction and insulin deficiency. ${ }^{1,3}$ As type 2 diabetes is a chronic disease that is aging-related, blood glucose control in many patients often requires the use of multipharmacological agents. Hence, there is always a need to develop novel pharmacological agents or chemicals that can be used to help fighting diabetes.

\section{5-methoxyindole-2-carboxylic acid as a hypoglycemic agent}

During our studies of mitochondrial dysfunction in stroke injury, we stumbled upon a chemical called 5-methoxyindole-2-carboxylic acid (MICA) (Figure 1), which exhibits a potential role in neuroprotection against stroke injury when applied as either a preconditioning or postconditioning agent. ${ }^{4,5} \mathrm{MICA}$ is often regarded as a biomarker for malignant melanoma, ${ }^{6}$ but intriguingly, a quick literature search indicates that MICA is actually also a hypoglycemic agent. ${ }^{7,8}$ In fact, this chemical, having an indole nucleus core structure that is known to possess antidiabetic properties, ${ }^{9,10}$ was studied
Correspondence: Liang-Jun Yan Department of Pharmaceutical Sciences, UNT System College of Pharmacy, University of North Texas Health Science Center, 3500 Camp Bowie Blvd, Fort Worth, TX 76107, USA

Tel + I 8177352386

Fax +l 8177352603

Email liang-jun.yan@unthsc.edu 
extensively in 1960s and 1970s in terms of its glucoselowering ability. It was found that MICA could inhibit liver gluconeogenesis by blocking carboxylation of pyruvate, ${ }^{11-14}$ a key reaction in the gluconeogenic pathway (Figure 2). The mechanism of this inhibition remains unknown as MICA was found to have no inhibitory effect on purified pyruvate carboxylase when incubated with the enzyme in vitro. ${ }^{14}$

MICA is also able to inhibit aerobic glucose degradation. The mechanism by which this works is that MICA can inhibit mitochondrial dihydrolipoamide dehydrogenase (DLDH), ${ }^{14-16}$ a component in the pyruvate dehydrogenase complex ${ }^{17,18}$ (Figure 3). This inhibition is reversible and can be reversed by lipoic acid, ${ }^{14}$ a cofactor for DLDH. It should be noted that MICA inhibition of the conversion of pyruvate to acetyl-CoA occurs not only just in the liver but also in many other tissues such as adipose, testis, brain, and kidney. ${ }^{5,12,19,20}$ The establishment that MICA inhibits DLDH is based on the evidence that the cofactor lipoic acid instead of other cofactors such as thiamine and thiamine pyrophosphate can reverse MICA inhibition of DLDH. ${ }^{14}$

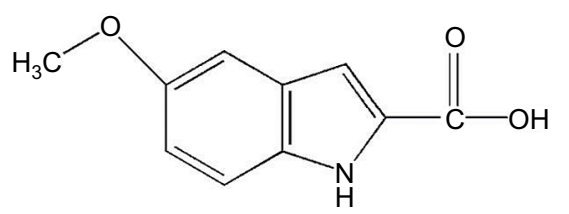

Figure I The chemical structure of MICA.

Abbreviation: MICA, 5-methoxyindole-2-carboxylic acid.

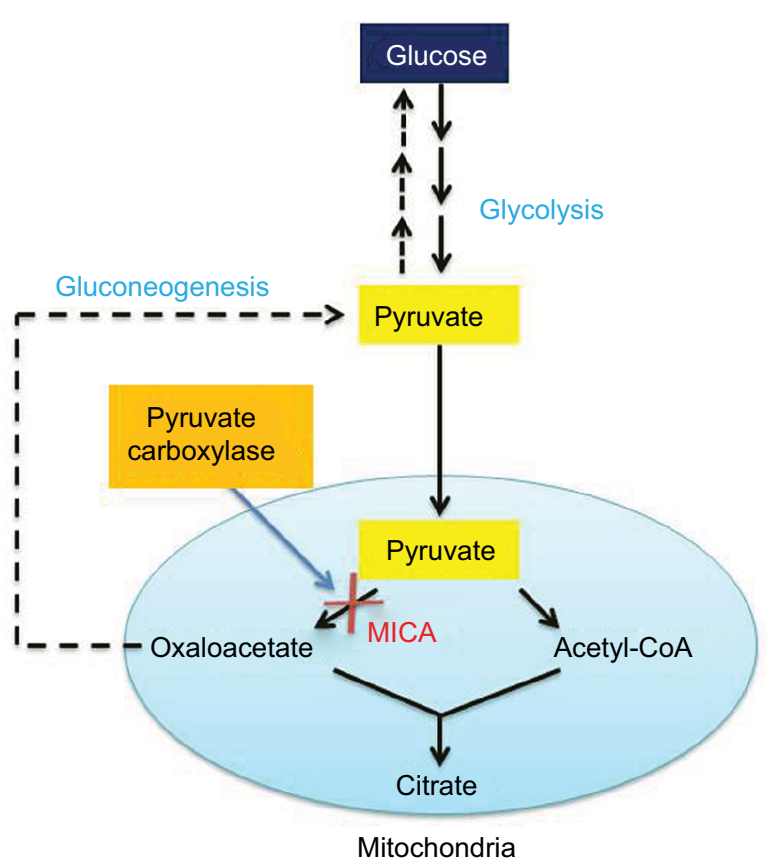

Figure 2 Diagram showing MICA block of pyruvate conversion to oxaloacetate in the gluconeogenesis pathway.

Abbreviation: MICA, 5-methoxyindole-2-carboxylic acid.
While the concept that MICA can exert its hypoglycemic effect via inhibition of the gluconeogenic pathway is straightforward, whether MICA can decrease blood glucose via inhibition of DLDH remains to be evaluated. Conceptually, when MICA blocks oxidation of pyruvate to acetyl-CoA, pyruvate would accumulate and more glucose would be consumed for ATP production by the glycolytic pathway in order to meet the body's energy needs. This would be similar to one of the mechanisms by which metformin increases glucose consumption by the glycolytic pathway via inhibiting mitochondrial complex I, leading to lowering of blood glucose. ${ }^{21}$ Therefore, MICA may also be able to lower blood glucose by inhibiting DLDH in diabetic subjects.

\section{MICA's metabolic effects and mortality rate in one diabetic animal model}

The study of MICA's hypoglycemic effect in the 1970s perhaps culminated by a short report that diabetic Chinese hamsters, when treated by MICA $(100 \mathrm{mg} / \mathrm{kg}$ body weight, via gavage) for approximately a week, showed a depressed blood glucose levels but also died at a greater rate than did control animals. ${ }^{22}$ The conclusion of this study was that while MICA could lower blood glucose in diabetic Chinese

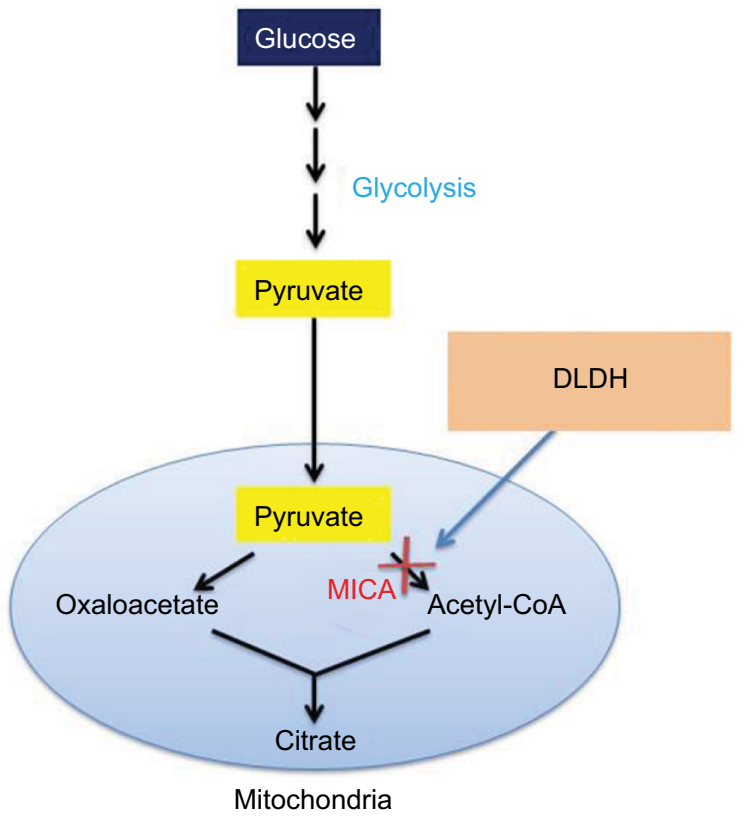

Figure 3 Diagram showing MICA block of pyruvate oxidation to acetyl-CoA in glucose combustion pathway.

Notes: MICA is known to physically bind to DLDH and exerts its inhibitory effect. It is also known that MICA binding to DLDH is a reversible process.

Abbreviations: DLDH, dihydrolipoamide dehydrogenase; MICA, 5-methoxyindole2-carboxylic acid. 
hamsters, it might not be a good antidiabetic agent because it increased the death rate of the treated diabetic animals. Since then, the property of MICA as a hypoglycemic agent has never been evaluated again, and most studies involving the use of MICA have been focused on its function as a DDH inhibitor. ${ }^{20,23,24}$ It should be pointed out that in this short and limited study that tested the hypoglycemic effect of MICA on diabetic Chinese hamsters, only one dosage and one means of MICA administration (via gavage) were tested. ${ }^{22}$ It should also be noted that this single study was very limited in scope and probably also dampened any further interest in studying MICA as a therapeutic drug for diabetes. In my opinion, such a single study is not enough to draw a conclusion that MICA is not a useful tool for managing diabetes. In particular, we and others have demonstrated that different administration routes could be important to minimize MICA's toxic effects and get beneficial results. ${ }^{4,5,7,8}$

\section{Perspectives}

Based on the above discussions that MICA is a potent inhibitor of the gluconeogenic pathway in the liver and is also a robust inhibitor of mitochondrial DLDH (as summarized in Figure 4), I think MICA should be further tested in many other rodent models of diabetes for its potential ability in lowering blood glucose. For a given diabetes model, the

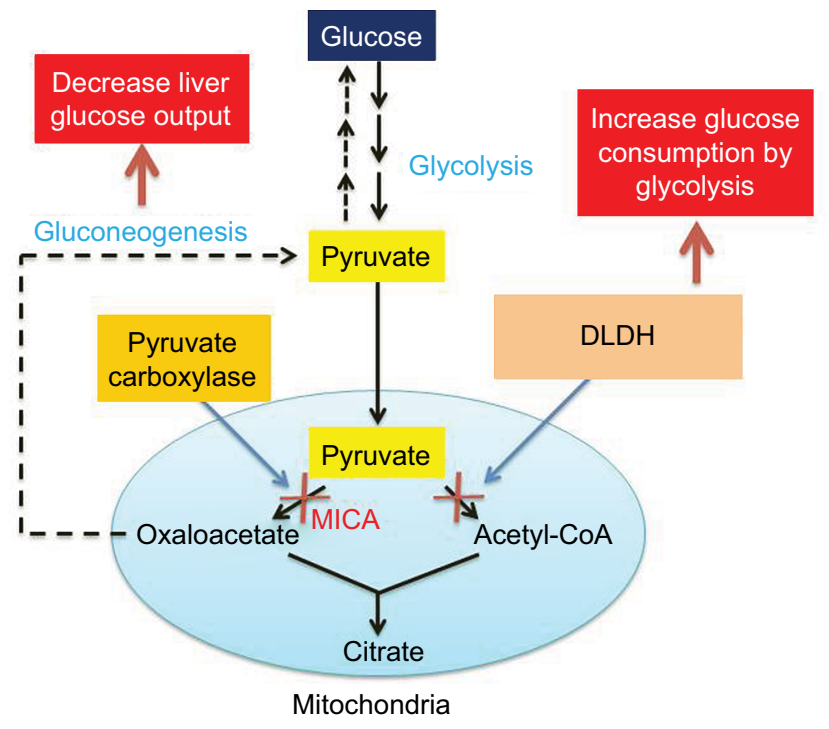

Figure 4 Scheme combined from Figures 2 and 3 showing the potential mechanisms by which MICA lowers blood glucose.

Notes: On one hand, MICA inhibits the gluconeogenic pathway in the liver resulting in a decrease in liver glucose output. On the other hand, MICA inhibits DLDH, decreasing ATP production by mitochondrial oxidative phosphorylation, thereby increasing aerobic glycolytic ATP production by consuming more glucose which could also lower blood glucose.

Abbreviations: DLDH, dihydrolipoamide dehydrogenase; MICA, 5-methoxyindole2-carboxylic acid. hypoglycemic effect of MICA and its toxic effect should be tested with different dosages in combination with different routes of administration. It may also be tested at different dosages with well-known antidiabetic drugs such as metformin and acarbose for its potential synergistic effect. In this regard, its tissue distribution and whether or how it is metabolized in the body should also be investigated.

Additionally, as MICA targets nearly all tissues in the body and has a variety of effects on protein expression and cellular pathways in numerous experimental settings or disease models, ${ }^{4,5,12,15,20,25-27}$ its potential effect on pancreatic $\beta$-cell function and insulin secretion in the context of diabetes should also be investigated. Moreover, a comprehensive evaluation of MICA on glucose metabolism, in particular, gluconeogenesis in cultured hepatocytes should also be performed.

Finally, it should be noted that in archaebacterium Haloferax volcanii that lacks lipoic acid in its less complex pyruvate dehydrogenase complex, ${ }^{28} \mathrm{MICA}$ is a potent inhibitor of the bacterial potassium ion transport and respiration, ${ }^{29}$ suggesting that MICA has other biological targets that could bear the toxic brunt of MICA while yielding overall hypoglycemic effects. In fact, in animal models, MICA has been shown to be able to target D-amino acid oxidase, though the inhibitory efficacy is very low. ${ }^{30}$ Therefore, it would not be surprising if MICA is found to target other proteins, which may also be implicated for its hypoglycemic function.

\section{Conclusion}

MICA, as a potential hypoglycemic agent, should be comprehensively reevaluated using a variety of diabetic animal models. In particular, its effect on mitochondrial bioenergetics, its pharmacokinetics and pharmacodynamics, its adverse effects, and its mechanisms of action on lowering blood glucose should all be studied. Moreover, its synergistic effect, if any, with other antidiabetic drugs, should also be investigated. Additionally, derivatives of MICA exhibiting decreased adverse effects may also be explored. MICA may well be a promising agent for fighting diabetes until proven otherwise.

\section{Acknowledgment}

This publication was supported in part by the National Institute of Neurological Disorders and Stroke (Grant number: R01NS079792) and by UNTHSC intramural grants RI10015 and RI10039.

\section{Disclosure}

The author reports no conflicts of interest in this work. 


\section{References}

1. Barnett AH. Type 2 Diabetes. 2nd ed. Oxford, UK: Oxford University Press; 2012.

2. Yan LJ. Pathogenesis of chronic hyperglycemia: from reductive stress to oxidative stress. J Diabetes Res. 2014;2014:137919.

3. Luo X, Wu J, Jing S, Yan LJ. Hyperglycemic stress and carbon stress in diabetic glucotoxicity. Aging Dis. 2016;7(1):90-110.

4. Wu J, Jin Z, Yang X, Yan LJ. Post-ischemic administration of 5-methoxyindole-2-carboxylic acid at the onset of reperfusion affords neuroprotection against stroke injury by preserving mitochondrial function and attenuating oxidative stress. Biochem Biophys Res Commun. 2018;497(1):444-450.

5. Wu J, Li R, Li W, et al. Administration of 5-methoxyindole-2-carboxylic acid that potentially targets mitochondrial dihydrolipoamide dehydrogenase confers cerebral preconditioning against ischemic stroke injury. Free Radic Biol Med. 2017;113:244-254.

6. Karnell R, von Schoultz E, Hansson LO, Nilsson B, Arstrand K, Kagedal B. S100B protein, 5-S-cysteinyldopa and 6-hydroxy-5-methoxyindole2-carboxylic acid as biochemical markers for survival prognosis in patients with malignant melanoma. Melanoma Res. 1997;7(5):393-399.

7. Hanson RL, Ray PD, Walter P, Lardy HA. Mode of action of hypoglycemic agents. I. Inhibition of gluconeogenesis by quinaldic acid and 5-methoxyindole-2-carboxylic acid. $J$ Biol Chem. 1969;244(16):4351-4359.

8. Reed J, Lardy HA. Mode of action of hypoglycemic agents. 3. Studies on 5-methoxy indole-2-carboxylic acid and quinaldic acid. J Biol Chem. 1970;245(20):5297-5303.

9. Li YY, Wu HS, Tang L, et al. The potential insulin sensitizing and glucose lowering effects of a novel indole derivative in vitro and in vivo. Pharmacol Res. 2007;56(4):335-343.

10. Sharma V, Kumar P, Pathak D. Biological importance of the indole nucleus in recent years: a comprehensive review. J Heterocycl Chem. 2010;47:491-502.

11. Daligcon BC, Oyama J, Hannak K. Increased gluconeogenesis in rats exposed to hyper-G stress. Life Sci. 1985;37(3):235-241.

12. Garcia-Salguero L, Aranda F, Peragon J, Corpas FJ, Lupianez JA. Metabolic adaptation of renal carbohydrate metabolism. IV. The use of site-specific liver gluconeogenesis inhibitors to ascertain the role of renal gluconeogenesis. Arch Int Physiol Biochim Biophys. 1991;99(3):237-242.

13. Bauman N, Pease BS. Effects of 5-methoxyindole-2-carboxylic acid on carbohydrate metabolism. Biochem Pharmacol. 1969;18(5):1093-1101.

14. Bauman N, Hill CJ. Inhibition of gluconeogenesis and $\alpha$-keto oxidation by 5-methoxyindole-2-carboxylic acid. Biochemistry. 1968;7(4):1322-1327.

15. Haramaki N, Han D, Handelman GJ, Tritschler HJ, Packer L. Cytosolic and mitochondrial systems for NADH- and NADPH-dependent reduction of $\alpha$-lipoic acid. Free Radic Biol Med. 1997;22(3):535-542.
16. Miller JA, Runkle SA, Tjalkens RB, Philbert MA. 1,3-Dinitrobenzene induced metabolic impairment through selective inactivation of the pyruvate dehydrogenase complex. Toxicol Sci. 2011;122(2):502-511.

17. Williams $\mathrm{CH}$ Jr. Lipoamide dehydrogenase, glutathione reductase, thioredoxin reductase, and mercuric ion reductase-a family of flavoenzyme transhydrogenases. In: Muller F, editor. Chemistry and Biochemistry of Flavoenzymes. Vol III. Boca Raton, FL, USA: CRC Press; 1992:121-212.

18. Yan LJ, Yang SH, Shu H, Prokai L, Forster MJ. Histochemical staining and quantification of dihydrolipoamide dehydrogenase diaphorase activity using blue native PAGE. Electrophoresis. 2007;28(7):1036-1045.

19. Gorin E, Zendowski S. Effect of methoxyindole 2-carboxylic acid and 4-pentenoic acid on adipose tissue metabolism. Biochim Biophys Acta. 1975;388(2):268-278.

20. Siva AB, Panneerdoss S, Sailasree P, Singh DK, Kameshwari DB, Shivaji S. Inhibiting sperm pyruvate dehydrogenase complex and its E3 subunit, dihydrolipoamide dehydrogenase affects fertilization in Syrian hamsters. PLoS One. 2014;9(5):e97916.

21. Andrzejewski S, Gravel SP, Pollak M, St-Pierre J. Metformin directly acts on mitochondria to alter cellular bioenergetics. Cancer Metab. 2014;2:12.

22. Schillinger $\mathrm{E}$, Loge $\mathrm{O}$. Metabolic effects and mortality rate in diabetic Chinese hamsters after long-term treatment with 5-methoxyindole2-carboxylic acid (MICA). Arzneimittelforschung. 1976;26(4):554-556.

23. Kumar V, Kota V, Shivaji S. Hamster sperm capacitation: role of pyruvate dehydrogenase A and dihydrolipoamide dehydrogenase. Biol Reprod. 2008;79(2):190-199.

24. Yan LJ, Thangthaeng N, Sumien N, Forster MJ. Serum dihydrolipoamide dehydrogenase is a labile enzyme. $J$ Biochem Pharmacol Res. 2013;1(1):30-42.

25. Richarme G, el Yaagoubi A, Kohiyama M. The MglA component of the binding protein-dependent galactose transport system of Salmonella typhimurium is a galactose-stimulated ATPase. $J$ Biol Chem. 1993;268(13):9473-9477.

26. Sun Z, Park SY, Hwang E, et al. Thymus vulgaris alleviates UVB irradiation induced skin damage via inhibition of MAPK/AP-1 and activation of Nrf2-ARE antioxidant system. J Cell Mol Med. 2017;21(2):336-348.

27. Aomine M. Effects of 5-methoxyindole-2-carboxylic acid on growth, glucose uptake and glycogen content in Tetrahymena pyriformis GL. Int J Biochem. 1977;8(1):27-32.

28. Kerscher L, Oesterhelt D. Pyruvate: ferredoxin oxidoreductase - new findings on an ancient enzyme. Trends Biochem Sci. 1982;7(10): 371-374.

29. Meury J. 5-Methoxyindole-2-carboxylic acid is a potent inhibitor of respiration and potassium ion transport in the archaebacterium Haloferax volcanni. FEMS Micobiol Lett. 1993;108(3):271-274.

30. Heefner DL, Currie MG, Rossi RF, Zepp CM, inventors; Sepracor Inc., assignee. D-amino acid oxidase inhibitors for learning and memory. United States patent US 20030162825A1. 2003.

Diabetes, Metabolic Syndrome and Obesity: Targets and Therapy

Dovepress

\section{Publish your work in this journal}

Diabetes, Metabolic Syndrome and Obesity: Targets and Therapy is an international, peer-reviewed open-access journal committed to the rapid publication of the latest laboratory and clinical findings in the fields of diabetes, metabolic syndrome and obesity research. Original research, review, case reports, hypothesis formation, expert opinion and commentaries are all considered for publication. The manuscript management system is completely online and includes a very quick and fair peer-review system, which is all easy to use. Visit http://www.dovepress.com/testimonials.php to read real quotes from published authors. 